\title{
Short-term results of a novel management of supracondylar fracture with coexisting osteoarthritis with bifold fixation and total knee arthroplasty
}

\author{
Nicholas A. Antao ${ }^{1}$, Sanjay Londhe ${ }^{1}$, Rajan Toor ${ }^{1^{*}}$ (D), Rajesh Shirishkar ${ }^{1}$ and Siddharth Aiyer $^{2}$
}

\begin{abstract}
Purpose: Presence of supracondylar and periarticular femoral fracture with associated arthritis of knee poses a challenging situation to the orthopaedic surgeon. The results of fixation of fracture in osteoporosis are not very satisfactory and have complications. With fixation alone, they still cannot bear weight on affected leg due to severe disability of osteoarthritis. To make patient walk, conventionally three surgeries in the form of fracture fixation, removal of implant and total knee arthroplasty (TKA) needs to be done in staged manner. We propose a novel management in form of bifold fixation and simultaneous TKA.
\end{abstract}

Methods: Eight cases ( 6 females, 2 males) of supracondylar femoral fractures with severe osteoarthritis of the knee and osteoporosis were primarily fixed with bifold fixation using SIGN nail (www.signfracturecare.org) and locking plate together with simultaneous total knee arthroplasty. There were five cases ( 2 males and 3 females) of grade 4 (Kellgren-Lawrence grading) osteoarthritis (OA) and three cases (all females) of severe rheumatoid arthritis (RA).

Results: The mean age was 68 years and average time for full weight bearing was 6 days. Radiographic evidence of fracture union was achieved in 16.25 weeks. The mean Knee Society Score (KSS) and Western Ontario and McMaster Universities Osteoarthritis Index (WOMAC) score at 26 months was 83.13 and 22.13 respectively.

Conclusions: Single stage combined bifold osteosynthesis with interlocking nail and locking plate together with total knee arthroplasty helps in one time management of these difficult injuries. It is a cost-effective and economically sound option and gives excellent results with good patient satisfaction.

Keywords: Total knee Arthroplasty, Supracondylar femoral fractures, Retrograde intramedullary solid locking nail (SIGN), Locking plate

\section{Introduction}

Distal femoral fractures in the elderly are a major cause of morbidity and mortality $[1,2]$. The reported incidence of distal femoral fractures is $6 \%$. However, they have 1-year mortality rates ranging between 15 and $40 \%$, similar to

*Correspondence: dr.rajantoor@gmail.com

${ }^{1}$ Department of Orthopaedics Holy Spirit Hospital, Jeevan Data Hostel, Mahakali Caves Road, Andheri (E), Mumbai, Maharashtra 400093, India

Full list of author information is available at the end of the article that of peri-trochanteric fractures [3-5]. These elderly patients often have concomitant secondary osteoarthritis of the knee joint, which impacts the eventual clinical and functional outcome [6,7]. The coexistent osteoporosis may lead to considerable comminution at the fracture site with intra-articular, metaphyseal or diaphyseal extension [8].

Traditionally, distal femoral fractures have been treated with osteosynthesis $[9,10]$. There has been a original author(s) and the source, provide a link to the Creative Commons licence, and indicate if changes were made. The images or other third party material in this article are included in the article's Creative Commons licence, unless indicated otherwise in a credit line to the material. If material is not included in the article's Creative Commons licence and your intended use is not permitted by statutory regulation or exceeds the permitted use, you will need to obtain permission directly from the copyright holder. To view a copy of this licence, visit http://creativecommons.org/licenses/by/4.0/. 
considerable evolution with time in the implants used for fixation. Blade plate and dynamic condylar screw with plate designs have given way more recently, and minimal invasive locking compression plates and retrograde distal femoral nails have become the preferred option for fixation [9-13]. Although these fixation techniques and devices give satisfactory results in younger individuals (less than 65 years), in the elderly (more than 65 years), achieving a stable fixation is challenging due to osteoporotic bone $[7,8,14]$. Even with a stable fixation, early weight bearing is not possible and partial weight bearing restrictions are difficult to execute for the elderly $[7,8]$. Often presence of other comorbidities, poor cognitive function, movement disorders or neurological deficit can make early mobilization practically impossible $[6,8]$. Prolonged recumbency in elderly patients has risk of further respiratory complications, pressure sores and eventually increase in morbidity and mortality $[6,15]$.

Primary arthroplasty has been increasingly used as a definitive management option for elbow, shoulder and hip trauma $[16,17]$. The proponents of this management strategy cited benefits including immediate stability, return of joint movement and early restoration of ambulatory function $[6,7,18]$.

The preliminary reports of the primary arthroplasty for the distal femoral fractures were made by Wolfgang et al and Bell et al in patients with rheumatoid arthritis and pre-existing osteoarthritis of the knee with a distal femoral fracture respectively $[19,20]$. Since then, many authors have reported small series of primary arthroplasty for proximal tibia/distal femoral fractures in the elderly with promising results $[7,21]$. The advantages quoted by these reports included early return to weight bearing and this strategy avoids a tedious reconstruction in the presence of a poor bone stock $[6,7,18,22]$. However, often these series have used special prosthesis, including constrained designs, long stemmed femoral/ tibial components and even modular tumour prosthesis $[6,8,15,21,22]$.

The authors reported a short-term outcomes of a unique surgical strategy for the treatment of distal femoral fractures in elderly patients with coexistent arthritis. This strategy combines the concept of a stable fixation achieved with a distal femoral locking compression plate over a distal femoral interlocking intramedullary nail. This fixation is then supplemented by replacement of the articulating surfaces with a cruciate-sacrificing TKA prosthesis.

\section{Material and methods}

A retrospective review of medical records was performed for eight patients undergoing distal femur fracture stabilization with the unique surgical strategy combining fracture fixation with replacement of the articular surfaces. The study was approved by the institutional review board and all ethical standards as per the Helsinki declaration and later amendments. Informed consent was obtained from all patients.

Inclusion criteria included was patients with B1 and B2 type supracondylar fracture with coexistent OA. Patients with B1 \& B2 type supracondylar fracture without coexistent $\mathrm{OA}$ and patients with $\mathrm{C} 1$ \& $\mathrm{C} 2$ type supracondylar fracture with coexistent OA were excluded from the study (Fig. 1).

There were six women and two men with a mean age of 68 years (64-75years). All eight patients had a distal femur fracture with associated arthritis in the knee, including five patients with primary osteoarthritis and three patients with rheumatoid arthritis. Two patients were socially-dependent and needed assistive devices for ambulation prior to fall. They were predominantly household ambulators. Six patients were independent community ambulators without the need of assistive devices.

\section{Implant and prosthesis design}

All fractures were stabilized using a retrograde solid intramedullary locking SIGN nail (Richland, Washington, USA). Locking bolts were placed both proximally and distally when the distal fracture segment length was more than three times the diameter of the femur at the fracture site. If the distal fracture segment was smaller than the diameter of the fracture, no locking bolts were inserted into the distal femoral segment. Locking bolts were inserted into the proximal femoral segment in patients with osteoporosis and comminution to prevent collapse due to instability. A locking compression plate was inserted in all patients over the less comminuted surface, to offer better metaphyseal fracture segment stabilization. The TKA prosthesis used in all patients was a cruciate-sacrificing Zimmer Biomet AGC knee.

\section{Procedure}

The surgical procedure was performed with the use of a well-padded tourniquet. A midline approach was used to expose the joint and the fracture site. The hematoma at the fracture site was evacuated and antibiotic wash (vancomycin with normal saline) was used during the procedure.

The steps of the surgical technique were as follows: (1) Temporary fracture reduction with reduction clamps which was confirmed on C-arm; (2) The prerequisite tibial, femoral and patellar cuts were taken and trial reduction was performed; (3) Trial implants were removed and retrograde intramedullary nailing was performed (Fig. 2) by using a solid SIGN nail of appropriate diameter ( 8 or $9 \mathrm{~mm}$ ). The nail of right length was selected so that 
SUPRACONDYLAR FEMUR FRACTURE ( $\mathrm{N}=28$ )
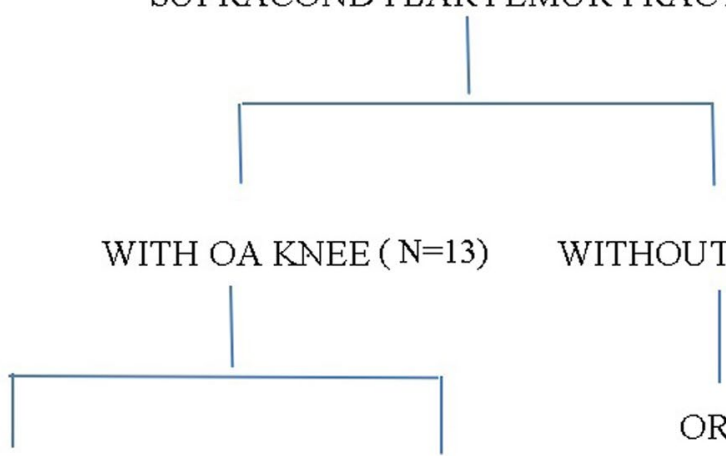

WITHOUT OA KNEE ( $\mathrm{N}=15)$

Type B1 and B2 fracture

Type $\mathrm{C} 1$ and $\mathrm{C} 2$ fracture

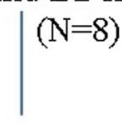

BIFOLD ORIF + TKA

Fig. 1 Flow chart of inclusion and exclusion criteria

the nail engages in the isthmus region of the femur; (4) A locking compression plate was applied to fix the metaphyseal region of the fracture with maximum alignment and stability; (5) TKA was performed by using a standard cruciate-sacrificing prosthesis without any constraint in the design.

\section{Postoperative protocol}

In the immediate postoperative period, patients were maintained in a long leg knee brace. They were put on physiotherapy as early as postoperative day one. Isometric exercise of the quadriceps and hamstrings, along with continuous passive mobilization of the knee joint was initiated. The range of motion was gradually increased based on pain tolerance to achieve a $90^{\circ}$ knee flexion by 2 to 4 weeks postoperatively as shown in Fig. 5 . Patients were encouraged to do partial weight-bearing as per their pain tolerance for a period of 4 to 10 days, followed by full weight bearing.

\section{Assessment of outcomes}

The clinical outcomes were assessed in terms of the range of motion achieved, time taken for weight bearing and length of hospital stay. The functional outcomes were assessed based on the Knee Society Scores (KSS) and the Western Ontario and Mc Master University Arthritis Score (WOMAC). WOMAC is a self-administered patient-reported outcome measure (PROM) consisting of 3 subscales, namely, pain (5 items), stiffness (2 items) and physical function (17 items). The test questions are scored on a scale of $0-4,0$ being none, 1 mild,

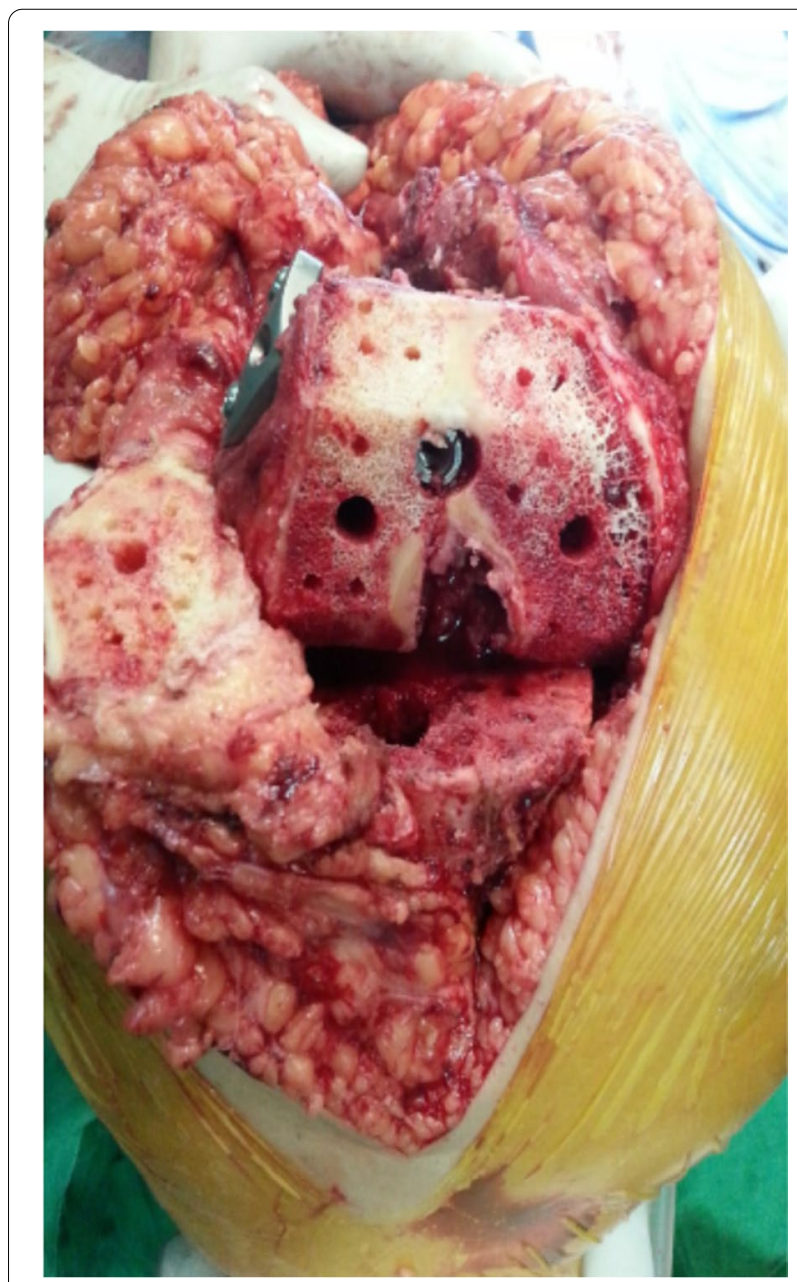

Fig. 2 The bone cuts with RIMN (SIGN nail) and locking plate 
Table 1 The preoperative, intraoperative and postoperative characteristics

\begin{tabular}{llllllllll}
\hline Case no. & Age (years) & Sex & Type & $\begin{array}{l}\text { Nail diameter } \\
(\mathbf{m m})\end{array}$ & $\begin{array}{l}\text { Weight } \\
\text { bearing (days) }\end{array}$ & KSS & WOMAC & $\begin{array}{c}\text { ROM (degrees) } \\
\text { Union } \\
\text { time } \\
\text { (weeks) }\end{array}$ \\
\hline 1 & 65 & Female & OA & 9 & 4 & 90 & 16 & 110 & 16 \\
2 & 64 & Female & OA & 9 & 10 & 80 & 24 & 100 & 17 \\
3 & 65 & Female & OA & 8 & 6 & 90 & 15 & 110 & 16 \\
4 & 70 & Male & RA & 8 & 4 & 65 & 42 & 90 & 16 \\
5 & 75 & Female & RA & 9 & 8 & 85 & 20 & 100 & 16 \\
6 & 72 & Female & OA & 9 & 6 & 90 & 14 & 105 & 16 \\
7 & 68 & Female & RA & 9 & 5 & 75 & 32 & 95 & 16 \\
8 & 65 & Male & OA & 8 & 7 & 90 & 14 & 100 & 17 \\
\hline
\end{tabular}

2 moderate, 3 severe and 4 extreme. Hence, the range of the score is from 0 to 96 . A higher WOMAC score indicates poor knee joint function and a lower score indicates good knee joint function. Radiographs were assessed for features of radiological union, coronal and sagittal alignment of the femoral and tibial components of the TKA. Survivorship of the TKA and any features of component loosening were noted. Mortality in the early perioperative period and 2-year mortality were recorded. Records were assessed for any complications occurring in the first 90-days follow-up and on subsequent follow-up for prosthetic joint infection and need of revision surgery.

\section{Results}

In our series of eight patients (Table 1), the mean duration of follow-up was 26 months (24-32 months). The mean length of stay in the hospital was 10 days and mean time to full weight-bearing mobilization was 6 days (range, 4-10days). There was no mortality in the eight patients in the 2-year follow-up.

Data including fracture classification, type of anaesthesia, surgical time, blood loss and units of blood transfusion are shown in Table 2.
Six patients who were community ambulators without the need of assistive devices were able to return to preinjury status. Two patients who were dependent household ambulators due to severe arthritis in opposite knee returned to pre-injury status.

Radiological evidence of fracture union was achieved in all patients in a mean duration of $16.25 \pm 0.46$ weeks (16-17 weeks). No radiological evidence of loosening of prosthesis was noted at final follow-up (Figs. 2 \& 3 ). The coronal and sagittal alignment of the femoral and tibial components were satisfactory in all patients. The clinical outcomes (Fig. 4) showed a mean range of knee flexion of $101.25^{\circ}\left(90-110^{\circ}\right)$. The mean KSS score was $83.13 \pm 9.23(65-90)$ and mean WOMAC score was $22.13 \pm 10.15$ (14-42). No complications such as surgical site infection, wound dehiscence, thromboembolic disease and prosthetic joint infection were noted Fig. 5.

\section{Discussion}

Chen et al noted in a systematic review that Type A fracture (extra-articular fracture) can be treated with long-stemmed femoral components with limited internal fixation to augment the stability [21]. Type B and

Table 2 Patient data of our series

\begin{tabular}{|c|c|c|c|c|c|}
\hline Case no. & $\begin{array}{l}\text { Fracture classification } \\
\text { (AO) }\end{array}$ & Type of anaesthesia & Surgery time & Blood loss & $\begin{array}{l}\text { Blood } \\
\text { transfusion } \\
\text { (Units of } \\
\text { packed cell } \\
\text { volume) }\end{array}$ \\
\hline 1 & Type B1 & Spinal+epidural & $2.30 \mathrm{~h}$ & $500 \mathrm{ml}$ & $1 \mathrm{PCV}$ \\
\hline 2 & Type B2 & Spinal+epidural & $2.35 \mathrm{~h}$ & $450 \mathrm{ml}$ & Nil \\
\hline 3 & Type B2 & Spinal+epidural & $2.45 \mathrm{~h}$ & $500 \mathrm{ml}$ & $1 \mathrm{PCV}$ \\
\hline 4 & Type B1 & Spinal+epidural & $2.30 \mathrm{~h}$ & $300 \mathrm{ml}$ & $\mathrm{Nil}$ \\
\hline 5 & Type B2 & Spinal+epidural & $2.30 \mathrm{~h}$ & $400 \mathrm{ml}$ & $\mathrm{Nil}$ \\
\hline 6 & Type B2 & Spinal+epidural & $2.50 \mathrm{~h}$ & $300 \mathrm{ml}$ & Nil \\
\hline 7 & Type B1 & Spinal+epidural & $2.45 \mathrm{~h}$ & $350 \mathrm{ml}$ & Nil \\
\hline 8 & Type B1 & Spinal+epidural & $2.40 \mathrm{~h}$ & $400 \mathrm{ml}$ & Nil \\
\hline
\end{tabular}




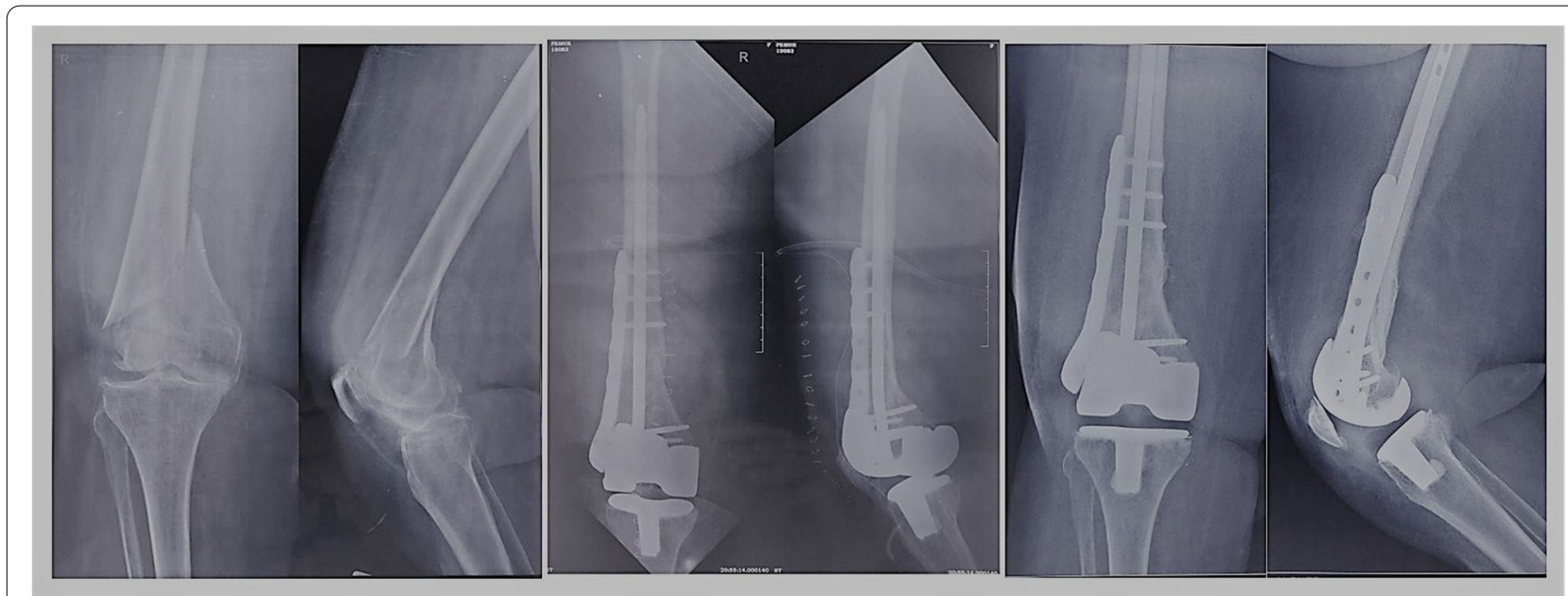

Fig. 3 a Preoperative distal femural fracture with OA knee. b Postoperative fracture fixation with TKA, nail and locking plate. c The healed fracture with implant in situ

type C fracture, which have intra-articular fracture extension, often need treatment with modular prosthesis or constrained design prosthesis based on the integrity of the collateral ligament attachments and extent of metaphyseal comminution [23]. The present series offers a unique approach where an unconstrained total arthroplasty prosthesis can be used provided a supplementary internal fixation is performed with an intramedullary solid nail and a locking compression plate at the same time.

Modular and constrained prostheses have a higher risk of aseptic loosening, mechanical failure and a lower survivorship compared to unconstrained TKA design in degenerative osteoarthritis $[6,8]$. The life expectancy of the patient must be taken into consideration when offering the constrained prosthesis/modular mega prosthesis to patients with distal femoral fractures and arthritis [8]. Parratte et al presented the only multicentre retrospective series analysing 26 patients operated in eight centres where unconstrained prosthesis was used in 9 patients, 12 patients needed medullary stem extenders and 5 patients had constrained hinge prostheses [24].

Mean range of knee flexion achieved in the present series was $101.25^{\circ}\left(90-110^{\circ}\right)$, which was comparable to the range achieved in other retrospective series by Nau et al, Wui et al and Boureau et al [18, 25, 26]. The mean KSS was 83.13 in our series and was comparable to the findings by

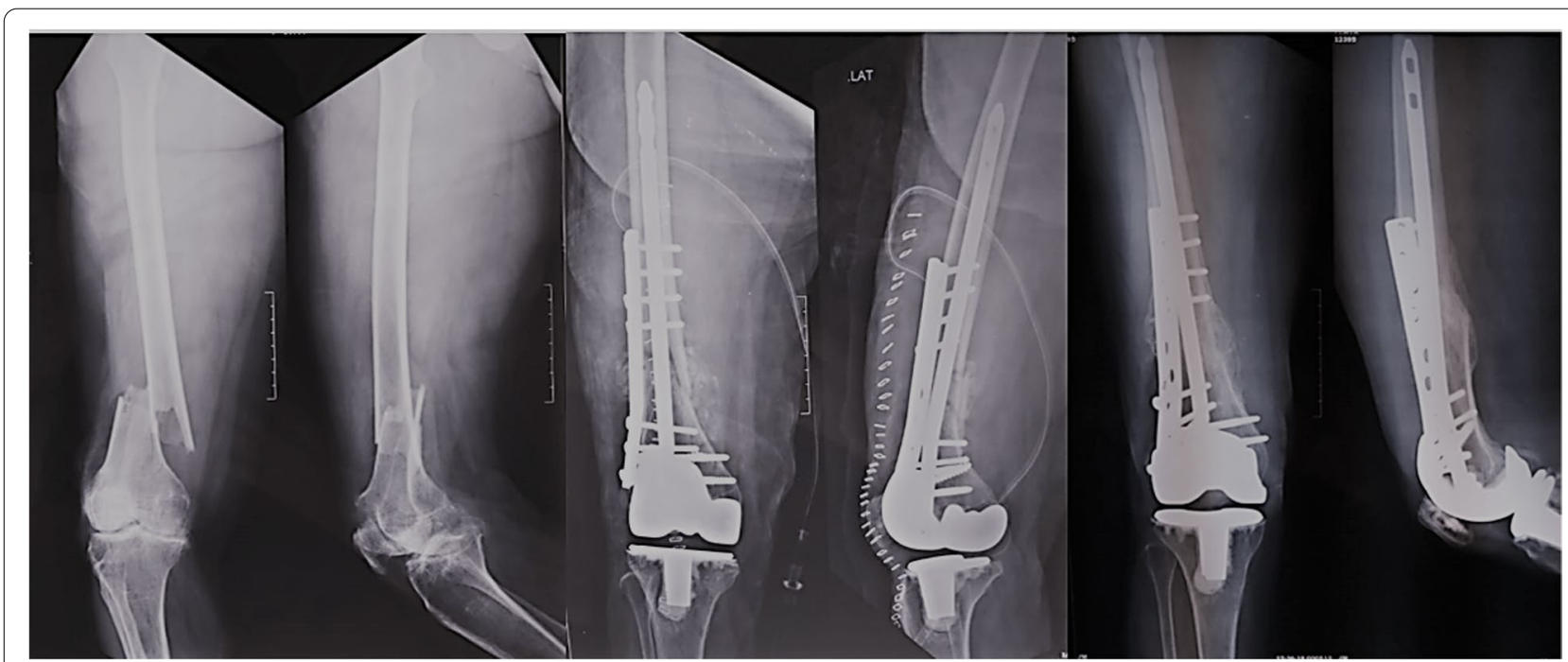

Fig. 4 a Preoperative distal femural fracture with RA knee. b Postoperative fracture fixation with TKA, nNail and locking plate. $\mathbf{c}$ Healed fracture with implants in situ 


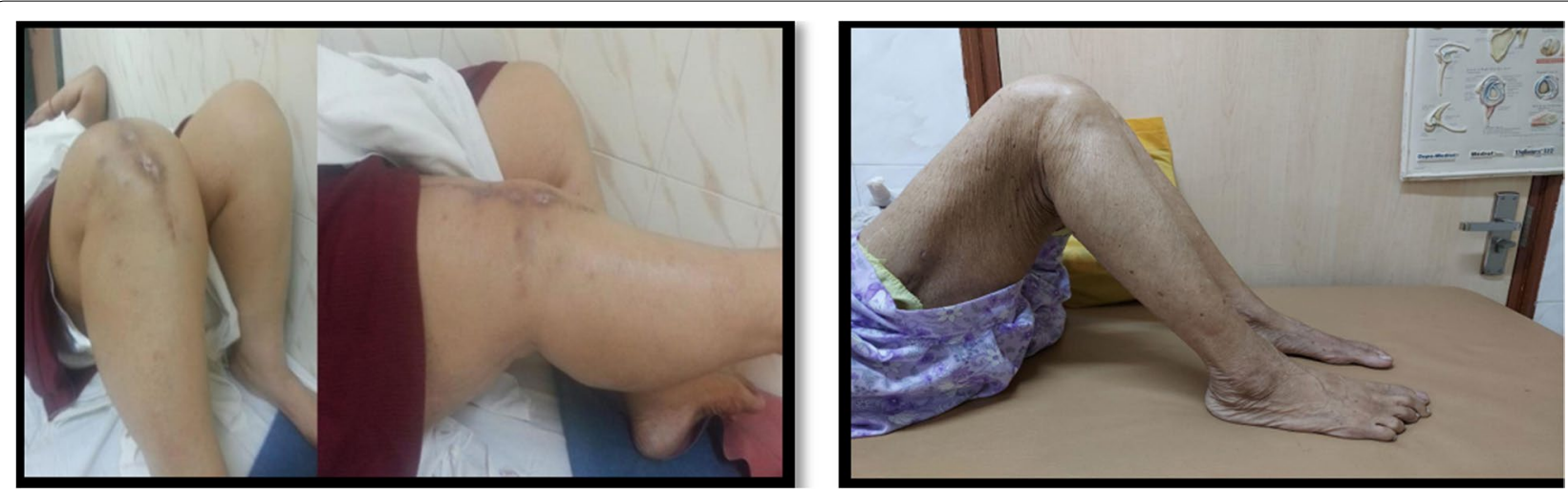

Fig. 5 a Full final function of a patient with distal femural fracture with OA knee. b Full final function of the patient with distal femural fracture with RA knee

Malviya et al, who reported a mean KSS of 90 in 26 elderly patients treated with primary arthroplasty for periarticular fractures [14]. Wui and co-workers documented a mean KSS of 87 in a series of 10 patients treated with arthroplasty following knee fractures at a 2-year follow-up [26].

Distal femoral fractures in the elderly have been associated with an increased 1-year mortality and in some series, have shown a rate comparable to that of hip fractures $[6,8$, $21]$. In our series, no mortality was noted in the first 2-year follow-up. However, Appleton and others reported one of the largest series of 52 patients of distal femoral fractures undergoing joint replacement procedures with a documented 1-year mortality of $42 \%$ [6]. The mean age of their cohort study was considerably higher ( 82 years) than the mean age of 68 years in our series.

One of the advantages of primary arthroplasty for distal femoral fractures is early return to weight bearing and ambulatory function [22, 23]. This is important for the elderly in whom prolonged recumbency is associated with thromboembolic events, pressure sores and respiratory complications [23]. Mean time to ambulation in our series was 6.25 days and passive knee range of movements were started within the first $48 \mathrm{~h}$. This early return to ambulatory function and no postoperative thromboembolic events were an encouraging outcome in the present series.

Reports by Appleton and Rosen have both documented early return to pre-injury ambulatory status after TKA in distal femoral fractures with arthritis [6, 22]. However, Boureau et al have reported a decline in the level of autonomy among the operated patients when compared with the pre-injury ambulatory function [18]. In our series, all the patients returned to pre-injury ambulatory status, including the two patients who needed walking aids even before injury.

In patients with intra-articular distal femoral fractures, a suboptimal reduction can lead to late post-traumatic arthritis [7, 23]. Outcomes of knee arthroplasty in the post-traumatic scenario are inferior to primary arthroplasty for degenerative arthritis [6, 27]. An additional procedure of implant removal is often necessary prior to embarking upon arthroplasty and surgical procedure may need to be staged with the use of constrained design prothesis, long-stemmed femoral component or hinged revision prostheses [23].

Our study has certain limitations. The first limitation is that the average age of the patients in our series was younger than that reported by Boureau et al and Paratte et $a l$. Hence, we are regularly following up these patients to see the long-term results of this novel technique. Another limitation of our study is that we did not compare this technique with the traditional method of management for these fractures, namely, open reduction \& internal fixation, then implant removal and the final third surgery in the form of TKA. We propose to do a formal prospective study comparing these two methods. Since we avoided two further surgeries, it reduced the total cost to the patient. The third limitation is that we used WOMAC score as the patient-reported outcome measure (PROM). This is in variance to the already described studies in which different outcome measures (PROM) were used.

One of the possible downsides of this technique is the theoretically increased risk of infection due to extra surgical time. However, we did not encounter infection in our patients.

These limitations are in line with most published literature on primary arthroplasty for distal femoral fractures $[7,21,23]$. We hereby presented the short-term outcomes of a unique strategy which, to the best of our knowledge, has not been previously reported.

\section{Conclusion}

In summary, the present series documented encouraging results with the use of a combination of surface replacement and bifold internal fixation for the 
treatment of distal femoral fractures with arthritis. These functional results were comparable with previously published literature on the use of primary arthroplasty for the treatment of distal femoral fractures. Using a bifold internal fixation has allowed for use of a standard TKA prosthesis without the need of any advanced prosthetic designs.

\section{Abbreviations}

TKA: Total Knee Arthroplasty; KL: Kellgren Lawrence; OA: Osteoarthritis; RA: Rheumatoid Arthritis; KSS: Knee Society Score; WOMAC: Western Ontario and McMaster Universities Osteoarthritis Index; RIMN: Rimmed Intramedullary Nail.

\section{Acknowledgements}

None.

\section{Authors' contributions}

Nicholas Antao: treatment, conceptualization, visualization, writing and revision of the original draft. Sanjay Londhe: writing and revision of the original draft. Rajan Toor: data collection, literature review and editing. Siddharth Aiyer: writing and revision of the original draft. Rajesh Shirishkar: writing and revision. All authors gave final approval to the version to be published, and agreed to be accountable for all aspects of the work.

\section{Availability of data and materials}

Transparent.

Funding: This research did not receive any specific grant from funding agencies in the public, commercial, or not-for-profit sectors.

\section{Declarations}

\section{Ethics approval and consent to participate}

Not required in view of retrospective study.

Written informed consent was obtained from the patients undergoing the surgery for use of their data and images without revealing their identity for scientific purpose.

\section{Competing interests}

None.

\section{Author details}

'Department of Orthopaedics Holy Spirit Hospital, Jeevan Data Hostel, Mahakali Caves Road, Andheri (E), Mumbai, Maharashtra 400093, India. ${ }^{2}$ Indian Orthopaedic Research Group, Thane, Maharashtra 400604, India.

Received: 14 June 2021 Accepted: 16 September 2021

Published online: 04 December 2021

\section{References}

1. Myers P, Laboe P, Johnson KJ, Fredericks PD, Crichlow RJ, Maar DC, et al. Patient mortality in geriatric distal femur fractures. J Orthop Trauma. 2018;32(3):111-5.

2. Elsoe R, Ceccotti AA, Larsen P. Population-based epidemiology and incidence of distal femur fractures. Int Orthop. 2018;42(1):191-6.

3. Court-Brown CM, Caesar B. Epidemiology of adult fractures: a review. Injury. 2006;37(8):691-7.

4. Martinet O, Cordey J, Harder Y, Maier A, Bühler M, Barraud GE. The epidemiology of fractures of the distal femur. Injury. 2000;31:62-94.

5. Streubel PN, Ricci WM, Wong A, Gardner MJ. Mortality after distal femur fractures in elderly patients. Clin Orthop Related Res. 2011;469(4):1188-96.

6. Appleton P, Moran M, Houshian S, Robinson CM. Distal femoral fractures treated by hinged total knee replacement in elderly patients. The journal of bone and joint surgery. Brit Vol. 2006;88(8):1065-70.
7. Bohm ER, Tufescu TV, Marsh JP. The operative management of osteoporotic fractures of the knee: to fix or replace? The journal of bone and joint surgery. Brit Vol. 2012;94(9):1160-9.

8. Ries MD. Primary arthroplasty for management of osteoporotic fractures about the knee. Curr Osteoporosis Reports. 2012:10(4):322-7.

9. Altenberg AR, Shorkey RL. Blade-plate fixation in non-union and in complicated fractures of the supracondylar region of the femur. JBJS. 1949;31(2):312-6.

10. Shewring DJ, Meggitt BF. Fractures of the distal femur treated with the AO dynamic condylar screw. The journal of bone and joint surgery. British volume. 1992;74(1):122-5.

11. Sanders R, Swiontkowski MA, Rosen H, Helfet D. Double-plating of comminuted, unstable fractures of the distal part of the femur. JBJS. 1991;73(3):341-6.

12. Danziger MB, Caucci D, Zecher SB, Segal D, Covall DJ. Treatment of intercondylar and supracondylar distal femur fractures using the GSH supracondylar nail. Am J Orthop. 1995;24(9):684-90.

13. Kregor PJ, Stannard JA, Zlowodzki M, Cole PA. Treatment of distal femur fractures using the less invasive stabilization system: surgical experience and early clinical results in 103 fractures. J Orthop Trauma. 2004;18(8):509-20.

14. Malviya A, Reed MR, Partington PF. Acute primary total knee arthroplasty for peri-articular knee fractures in patients over 65 years of age. Injury 2011;42(11):1368-71.

15. Choi NY, Sohn JM, Cho SG, Kim SC, In Y. Primary total knee arthroplasty for simple distal femoral fractures in elderly patients with knee osteoarthritis. Knee Surg Related Res. 2013;25(3):141.

16. Wretenberg $P$, Ekelund A. Acute hemiarthroplasty after proximal humerus fracture in old patients: a retrospective evaluation of 18 patients followed for 2-7 years. Acta Orthop Scand. 1997;68(2):121-3.

17. Morrey BF. Fractures of the distal humerus: role of elbow replacement. Orthop Clin North Am. 2000;31(1):145-54.

18. Boureau F, Benad K, Putman S, Dereudre G, Kern G, Chantelot C. Does primary total knee arthroplasty for acute knee joint fracture maintain autonomy in the elderly? A retrospective study of 21 cases. Orthop Traumatol Surg Res. 2015;101(8):947-51.

19. Wolfgang gl. Primary Total Knee Arthroplasty for Intercondylar Fracture of the Femur in a Rheumatoid Arthritic Patient: A Case Report. Clin Orthopaed Related Res. 1982; 171:80-2.

20. Bell KM, Johnstone AJ, Court-Brown CM, Hughes SP. Primary knee arthroplasty for distal femoral fractures in elderly patients. The journal of bone and joint surgery. Brit Vol. 1992:74(3):400-2.

21. Chen F, Li R, Lall A, Schwechter EM. Primary total knee arthroplasty for distal femur fractures: a systematic review of indications, implants, techniques, and results. Am J Orthop (Belle Mead NJ). 2017;46(3):E163-1.

22. Rosen AL, Strauss E. Primary total knee arthroplasty for complex distal femur fractures in elderly patients. Clin Orthopaed Related Res. 2004; 425:101-5.

23. Parratte S, Ollivier M, Argenson JN. Primary total knee arthroplasty for acute fracture around the knee. Orthop Traumatol Surg Res. 2018:104(1):S71-80

24. Parratte S, Bonnevialle P, Pietu G, Saragaglia D, Cherrier B, Lafosse JM. Primary total knee arthroplasty in the management of epiphyseal fracture around the knee. Orthop Traumatol Surg Res. 2011;97(6):S87-94.

25. Nau T, Pflegerl E, Erhart J, Vecsei V. Primary total knee arthroplasty for periarticular fractures. J Arthroplast. 2003;18(8):968-71.

26. Wui NB, Anuar MA, Wahid AM. Short-term outcome of early primary Total knee Arthroplasty for fractures around the knee in the elderly population: the experience of a secondary healthcare Centre in Malaysia. Malaysian J Med Sci. 2020:27(4):64.

27. Ebied A, Zayda A, Marei S, Elsayed H. Medium term results of total knee arthroplasty as a primary treatment for knee fractures. SICOT-J. 2018;4.

\section{Publisher's Note}

Springer Nature remains neutral with regard to jurisdictional claims in published maps and institutional affiliations. 